\title{
TRIVIAL CYCLES IN GRAPHS EMBEDDED IN 3-MANIFOLDS
}

\author{
YING-QING WU \\ University of Iowa, Iowa City, IA 52242
}

\begin{abstract}
A cycle $C$ of a graph $\Gamma$ embedded in a 3-manifold $M$ is said to be trivial in $\Gamma$ if it bounds a disk with interior disjoint from $\Gamma$. Let $e$ be an edge of $\Gamma$ with ends on $C$. We will study the relation between triviality of cycles in $\Gamma$ and that of $\Gamma-e$ and $\Gamma / e$. Let $C_{1}$ be one of the two cycles in $C \cup e$ containing $e$. The main theorem says that if $C$ is trivial in $\Gamma-e$ and $C_{1} / e$ is trivial in $\Gamma / e$, then either $C$ or $C_{1}$ is trivial in $\Gamma$. Some applications to cycle trivial graphs will be given in Section 2 .
\end{abstract}

\section{The Main Theorem}

If $L$ is a link in $S^{3}$, then a component $K$ of $L$ is said to be trivial if it bounds a disk in $S^{3}$ which is disjoint from the other components of $L$. This can be generalized to graphs in 3-manifolds. Recall that a cycle $C$ of a graph $\Gamma$ is an embedded circle. Suppose that $\Gamma$ is a graph in a 3 -manifold $M$. Then $C$ is called a trivial cycle in $\Gamma$ if it bounds a disk $D$ in $M$ with interior disjoint from $\Gamma$. Of course this depends on $\Gamma$ and the way it is embedded in $M$, so we may also say that $C$ is trivial with respect to $(M, \Gamma)$.

Suppose $e$ is a non loop edge in $\Gamma$. Then we have a subgraph $\Gamma-e$ in $M$, and a quotient graph $\Gamma / e$ in $M / e \cong M$. We are interested in the problem of how the triviality of cycles in $\Gamma$ is related to that of $\Gamma-e$ and $\Gamma / e$. If $e$ is disjoint from $C$, then clearly $C$ is trivial in $\Gamma$ if and only if it is trivial in $\Gamma / e$. When $e$ has one end on $C$ the problem was studied in [12], and it was shown that if $C$ is trivial in both $\Gamma / e$ and $\Gamma-e$, then it is trivial in $\Gamma$.

In this paper we study the case that $C \cap e=\partial e$. Let $C_{1}$ be one of the two cycles in $C \cup e$ that contain $e$. In this case $C / e$ is no longer a cycle, but $C_{1} / e$ is a cycle in $\Gamma / e$. Consider the following examples.

1991 Mathematics Subject Classification. Primary 57M15, secondary 57N10.

Key words and phrases. Trivial cycles, graphs, 3-manifolds. 
Example 1.1. Let $C$ be the cycle $e_{1} \cup e_{2}$ in Figure 1. $C$ is trivial in $\Gamma-e$, but it is nontrivial in $\Gamma$.

Example 1.2. For the graph $\Gamma$ in Figure 2, let $C_{1}=e_{1} \cup e$. Then $C_{1} / e$ is trivial in $\Gamma / e$, but $C_{1}$ is nontrivial in $\Gamma$.

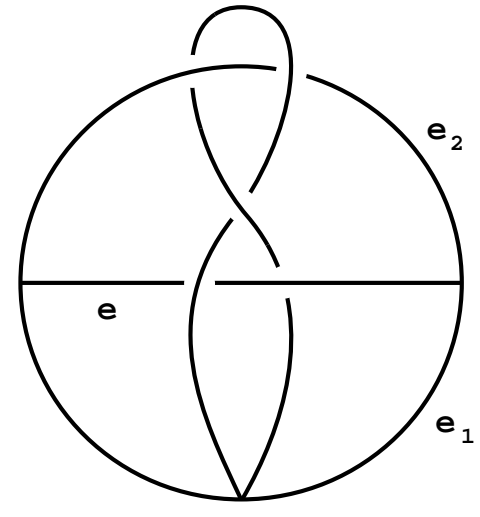

Figure 1

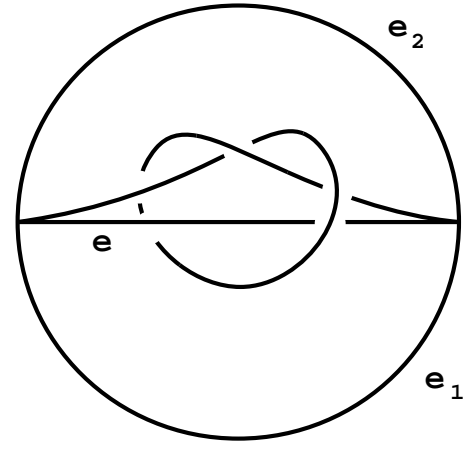

Figure 2

The main result of this paper is Theorem 1.4, which says that these two cases can not happen simultaneously: If $C$ is trivial in $\Gamma-e$, and $C_{1} / e$ is trivial in $\Gamma / e$, then either $C$ or $C_{1}$ has to be trivial in $\Gamma$. In this section we will prove this theorem. Some applications will be given in the next section.

Consider a graph $\Gamma$ in a 3-manifold $M$. Let $N(\Gamma)$ be a regular neighborhood of $\Gamma$. Define the exterior of $\Gamma$ to be $E(\Gamma)=M-\operatorname{Int} N(\Gamma)$. If $v_{1}, \ldots, v_{r}$ are the vertices of $\Gamma$, and $e_{1}, \ldots e_{t}$ the edges, then $N(\Gamma)$ is a union of $N\left(v_{i}\right)$ and $N\left(e_{j}\right)$, where $N\left(e_{j}\right)$ is chosen to be small enough so that each $N\left(e_{j}\right)$ intersects $\cup N\left(v_{i}\right)$ in two disks, and all the disks in $\left\{N\left(v_{i}\right) \cap N\left(e_{j}\right) \mid j=1, \ldots t\right\}$ are mutually disjoint. Let $\delta\left(v_{i}\right)$ be the punctured sphere $\partial N\left(v_{i}\right)-\cup \operatorname{Int} N\left(e_{j}\right)$, and let $\delta\left(e_{j}\right)$ be the annulus $\partial N\left(e_{j}\right)-\cup \operatorname{Int} N\left(v_{i}\right)$.

If $\Gamma^{\prime}$ is a subgraph of $\Gamma$, define $\delta\left(\Gamma^{\prime}\right)$ to be the union of $\delta(t)$, where $t$ ranges over all vertices and edges of $\Gamma^{\prime}$. In particular, if $C$ is a cycle, then $\delta(C)$ is a punctured torus. If $C$ is a trivial cycle in $\Gamma$, then it bounds a disk $D$ whose intersection with $E(\Gamma)$ is a disk $D^{\prime}$. The disk $D$ can be chosen so that $\partial D^{\prime} \subset \delta(C)$, and for each edge $e_{j}$ of $C$, $\partial D^{\prime}$ intersects $\delta\left(e_{j}\right)$ at exactly one essential arc. Conversely, if there is a disk $D^{\prime}$ in $E(\Gamma)$ satisfying these conditions, then $D^{\prime}$ can be extended to a disk $D$ in $M$ so that $D \cap \Gamma=\partial D=C$. Therefore we have

Lemma 1.3. A cycle $C$ in $\Gamma$ is trivial if and only if there is a disk $D^{\prime}$ in $E(\Gamma)$ so that $\partial D^{\prime} \subset \delta(C)$, and $\partial D^{\prime} \cap \delta\left(e_{j}\right)$ is an essential arc of $\delta\left(e_{j}\right)$ for all edges $e_{j}$ of $C$. 
Now let $e$ be a non loop edge of $\Gamma$. Consider the quotient graph $\Gamma / e$. We use $[e]$ to denote the image of $e$ in $\Gamma / e$. Suppose $e$ is incident to the vertices $v_{1}$ and $v_{r}$. We identify the regular neighborhood $N(\Gamma / e)$ with $N(\Gamma)$ by letting $N([e])=N(e) \cup N\left(v_{1}\right) \cup N\left(v_{r}\right)$. Under this identification we have $E(\Gamma)=E(\Gamma / e)$ and $\delta([e])=\delta(e) \cup \delta\left(v_{1}\right) \cup \delta\left(v_{r}\right)$.

Theorem 1.4. Let $C$ be a cycle in $\Gamma$. Let e be a non loop edge with $e \cap C=\partial e$. Let $C_{1}$ be one of the two cycles in $C \cup e$ that contain e. If $C$ is trivial in $\Gamma-e$, and $C_{1} / e$ is trivial in $\Gamma / e$, then either $C$ or $C_{1}$ is trivial in $\Gamma$.

Proof. Let $e_{1}, \ldots e_{n}$ and $v_{1}, \ldots, v_{n}$ be the edges and vertices of $C$, with $\partial e_{i}=v_{i} \cup v_{i+1}$, $\left(v_{n+1}=v_{1}\right)$. Without loss of generality we may assume that $\partial e=v_{1} \cup v_{r}$, and $C_{1}=e_{1} \cup \ldots \cup e_{r-1} \cup e$.

By assumption $C$ bounds a disk $D$ in $M$ with $\operatorname{Int} D \cap(\Gamma-e)=\emptyset$. Let $t_{1}, \ldots, t_{p}$ be the intersection points of $e \cap \operatorname{Int} D$, labeled successively along $e$. Choose $D$ so that $p$ is minimal. If $p=0$, then $D$ is disjoint from $e$ and we are done. So we assume that $p>0$.

The surface $P=D \cap E(\Gamma)$ is a punctured disk with boundary $\partial P=\partial_{0} \cup \ldots \cup \partial_{p}$, where $\partial_{i}(i \geq 1)$ is the boundary of the disk in $N(e) \cap D$ that contains $t_{i}$, and $\partial_{0}$ lies on $\delta(C)$, intersecting each of $\delta\left(e_{i}\right)$ and $\delta\left(v_{j}\right)$ at an essential arc. See Figure 3, where $p=2$.

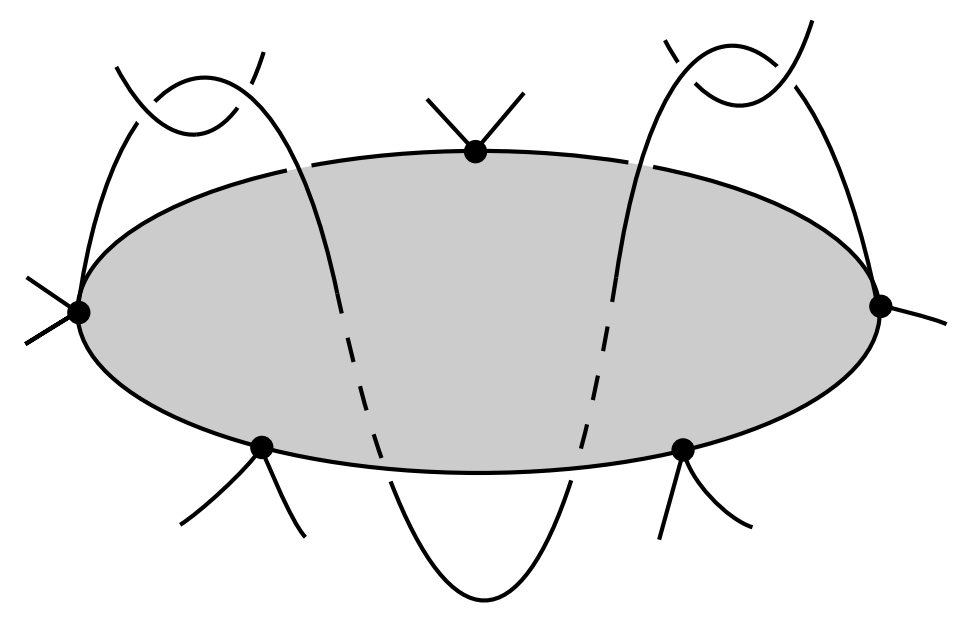

Figure 3

Now consider the cycle $C_{1} / e$ in $\Gamma / e$. By assumption $C_{1} / e$ is trivial in $\Gamma / e$, so it bounds a disk $D_{1}$ in $M / e \cong M$ whose interior is disjoint from $\Gamma / e$. The intersection of $D_{1}$ with $E(\Gamma)=E(\Gamma / e)$ is a disk $Q$. By Lemma 1.3 we may assume that $\partial Q \cap \delta\left(e_{i}\right)$ is an essential curve on $\delta\left(e_{i}\right)$ for $i=1, \ldots, r-1$. If $\partial Q \cap \delta(e)$ is also a single essential curve, then $Q$ extends to a disk in $M$ bounded by $C_{1}$, and we are done. Therefore we 
assume that $\partial Q \cap \delta(e)$ is a set of $q>1$ essential arcs, each intersecting $\partial_{i}$ at a single point for $i=1, \ldots, p$. The set $G=P \cap Q$ is a properly embedded 1-manifold in both $P$ and $Q$. Moreover, by an isotopy we may push all the ends of $G$ off the annulus $\delta\left(e_{i}\right)$, $i \geq 1$. Thus $\partial G \cap \partial_{0} \subset \cup \delta\left(v_{i}\right)$. Choose $P$ and $Q$ so that $|G|$ is minimal subject to the minimality conditions of $p$ and $q$. When considering $G$ as a subset of $P$, we denote it by $G_{P}$. Similarly for $G_{Q}$.

CLAIM 1: $G_{P}$ is a set of essential $\operatorname{arcs}$ in $P$.

Proof. This is essentially an innermost circle outermost arc argument. By surger $P$ along disks on $Q$ bounded by innermost circles of $G_{Q}$, we can eliminate all circle components of $G_{P}$. Similarly one can remove all trivial arcs of $G_{P}$ which has ends on $\partial_{i}$ with $i \geq 1$, by surger $Q$ along disks on $P$ cut off by such arcs.

We need to be a little careful when an outermost $\operatorname{arc} \gamma$ of $G_{P}$ has ends on $\partial_{0}$. Let $\Delta$ be the disk on $P$ cut off by $\gamma$. Let $Q_{1}, Q_{2}$ be the closure of the two components of $Q-\gamma$. The point here is that, since each of $\partial P$ and $\partial Q$ intersect $\delta\left(e_{i}\right)$ in just one essential arc, one of the $Q_{i}$, say $Q_{1}$, has the property that $\partial Q_{1}$ intersects some $\delta\left(e_{i}\right)$ if and only if $\partial \Delta$ does, so if we replace $Q_{1}$ by $\Delta$, the new disk $Q^{\prime}=Q_{2} \cup \Delta$ still has the property that it intersects each $\delta\left(e_{i}\right)$ just once for $i=1, \ldots, r-1$. We can thus replace $Q$ by $Q^{\prime}$ to reduce $|G|$.

Now consider the $\operatorname{arcs} G_{Q} \subset Q$. For each end $x$ of $G_{Q}$, define a label $l(x)$ as follows. If $x$ lies on $\partial_{i}$ with $i \geq 1$, define $l(x)=i$. If $x$ lies on $\delta\left(v_{j}\right)$, define $l(x)=-j$. Thus, when traveling along $\partial\left(Q_{1}\right)$, the labels appear as a string of the form

$$
(-1)^{n_{1}}(-2)^{n_{2}} \ldots(-r)^{n_{r}} p \ldots 21(-1)^{k_{1}} 12 \ldots p(-r)^{k_{2}} \ldots(-r)^{k_{q-1}} p \ldots 21,
$$

where $n_{i}, k_{j} \geq 0$.

CLAIM 2. If $\left\{l_{1}, l_{2}\right\}$ are the labels of an outermost arc $\gamma$ of $G_{Q}$, then $l_{1}=1$ or $p$, and $l_{2}$ is between -2 and $-r+1$. In particular, $n_{1}=n_{r}=0$, and all arcs of $G_{Q}$ are parallel, having at least one negative labels on its ends.

Proof. The labels of an outermost arc must be adjacent in the above label string, so $\left\{l_{1}, l_{2}\right\}$ has the following possibilities.

(1) $\quad\left\{l_{1}, l_{2}\right\}=\{i, i+1\}, i \geq 1$;

(2) $\left\{l_{1}, l_{2}\right\}=\{1,-1\}$ or $\{p,-r\}$;

(3) both $l_{i}$ are negative; 
(4) $\left\{l_{1}, l_{2}\right\}=\{1,1\}$ or $\{p, p\}$;

(5) $\left\{l_{1}, l_{2}\right\}=\{1, p\}$;

(6) $l_{1}=1$ or $p$, and $-r+1 \leq l_{2} \leq 2$.

Note that (4) happens only if some $k_{i}=0$, and (5) happens only if all $n_{i}=0$.

Cases (1) - (3) are easy to rule out. If $\left\{l_{1}, l_{2}\right\}=\{i, i+1\}$ for some $i \geq 1$, we can isotop the edge $e$ through the outermost disk $\Delta$ on $Q$ cut off by $\gamma$ to reduce $|e \cap D|$, contradicting the minimality of $p$. This also works if $\left\{l_{1}, l_{2}\right\}=\{1,-1\}$ or $\{p,-r\}$. (But it does not work if $\left\{l_{1}, l_{2}\right\}=\{1,-2\}$, say, because then part of $\partial \Delta$ lies on the cycle $C$.) If $\left\{l_{1}, l_{2}\right\}$ are both negative, then one can surger $P$ along $\Delta$ to obtain a new $P^{\prime}$ which has less intersection with $Q$. Note that $\partial P^{\prime}$ still intersects each $\delta\left(e_{j}\right)$ just once, so it can be used to replace $P$.

Now assume $\left\{l_{1}, l_{2}\right\}=\{1,1\}$. Since each positive label appears an odd number of times, some arc $\alpha$ of $G_{Q}$ has different labels on its ends. Let $\Delta$ be the component of $Q-\alpha$ that contains the outermost arc $\gamma$. We may choose $\alpha$ to be extremal in the sense that the two labels of each arc of $G_{Q}$ in $\Delta$ are the same. This means that each arc in $\Delta$ corresponds to a loop in $G_{P}$. If $p$ is a label of some arc in $\Delta$, then all positive labels appear in $\Delta$, so each vertex of $G_{P}$ has a loop based on it. But then one of these loops must be an inessential arc of $G_{P}$, contradicting claim 1. Therefore $p$ does not appear as a label of $\operatorname{arcs}$ in $\Delta$. It is now easy to see that $\gamma$ is the only outermost arc in $\Delta$, so all the arcs in $\Delta$ are parallel to each other. But this and the fact that $p$ does not appear on $\Delta$ imply that the labels of $\alpha$ are the same, contradicting the choice of $\alpha$. Therefore (4) can not happen.

If (5) happens, then all $n_{i}=0$, so no outermost arc can be of type (6). From the label string one can see that there can be at most one type (5) arc. If $G_{Q}$ has just one arc, (5) would be the same as (1), which has been ruled out. If $G_{Q}$ has more than one arcs, then it has at least two outermost arcs, so the ones other than $\gamma$ must be of types (1) - (4), which is impossible by the above. Therefore (5) can not happen either.

It follows that $\gamma$ must be of type (6). From the label string it is clear that there are at most two such outermost arcs. Therefore all arcs of $G_{Q}$ are parallel.

Now consider the curves in $G_{P}$. By assumption, for $i \geq 1, \partial Q$ intersect each $\partial_{i}$ exactly $q$ times, $q \geq 3$, so each $\partial_{i}$ is incident to $q \operatorname{arcs}$ of $G_{P}$. By Claim 2 , all of these arcs have the other ends on $\partial_{0}$. (Cf. Figure 4.) Therefore, there is a pair of parallel arcs $b_{1}, b_{2}$ on $G_{P}$, which is outermost in the sense that they cut off a disk $\Delta$ with interior disjoint from $Q$. Let $\Delta^{\prime}$ be the disk on $Q$ between $b_{1}, b_{2}$. Form a disk $Q^{\prime}=\left(Q-\Delta^{\prime}\right) \cup \Delta$. 
One can see that $Q^{\prime}$ can be isotoped to have less that $q$ intersection $\operatorname{arcs}$ with $\delta(e)$, and $\partial Q^{\prime}$ intersects $\delta\left(e_{j}\right)$ just once for $j=1, \ldots, r-1$. This contradicts the choice of $Q$, completing the proof of Theorem 1.4.

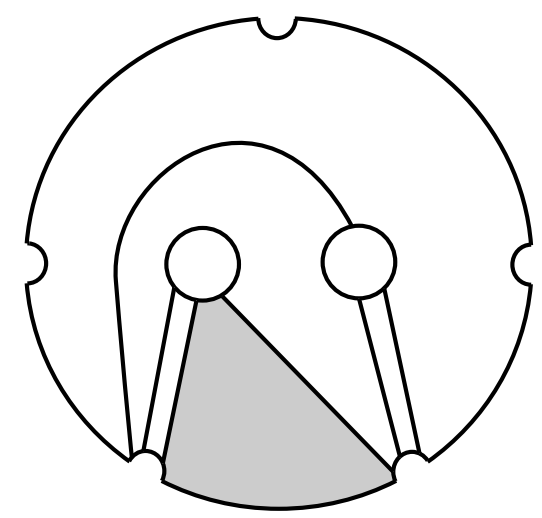

Figure 4

\section{Some Applications}

The following theorem was proved in [12].

Theorem 2.1. Suppose $\Gamma$ is a graph embedded in a 3-manifold $M$. Let $C$ be a cycle in $\Gamma$, and let $e$ be an edge of $\Gamma$ with at most one end on $C$. If $C$ is trivial in both $\Gamma-e$ and $\Gamma / e$, then it is trivial in $\Gamma$.

If $e$ has both ends on $C$, then $C / e$ is a union of two cycles. The following result has a similar nature as that above. The difference is that when considering the quotient graph, we need to consider both cycles in $C / e$.

Corollary 2.2. Suppose $\Gamma$ is a graph embedded in a 3-manifold $M$. Let $C$ be a cycle in $\Gamma$. Let e be a non loop edge of $\Gamma$ such that $\partial e=e \cap C$. If $C$ is trivial in $\Gamma-e$, and if the two cycles in $C / e$ are trivial in $\Gamma / e$, then $C$ is trivial in $\Gamma$.

Proof. Let $C_{1}, C_{2}$ be the two cycles in $C \cup e$ that contains e. By Theorem 1.4, either $C$ is trivial in $\Gamma$ and we are done, or both $C_{1}, C_{2}$ are trivial in $\Gamma$. Since $C_{1} \cap C_{2}=e$ is connected, by Lemma 1.1 of [12], $C_{i}$ bounds a disk $D_{i}$ with interior disjoint from $\Gamma$, such that $D_{1} \cap D_{2}=e$. Let $D$ be $D_{1} \cup D_{2}$ with interior pushed off $e$, then $D \cap \Gamma=C$, therefore $C$ is trivial in $\Gamma$.

A subgraph $\Gamma^{\prime}$ of $\Gamma$ is said to be cycle trivial in $\Gamma$ if all cycles of $\Gamma^{\prime}$ are trivial in $\Gamma$. When $\Gamma^{\prime}=\Gamma$, we simply say that $\Gamma$ is cycle trivial. The following corollary follows 
immediately from Theorem 2.1 and Corollary 2.2, by applying the results to each cycle of $\Gamma^{\prime}$.

Corollary 2.3. Let $\Gamma^{\prime}$ be a subgraph of $\Gamma$, and let e be a non loop edge of $\Gamma-\Gamma^{\prime}$. If $\Gamma^{\prime}$ is cycle trivial in $\Gamma-e$, and $\Gamma^{\prime} / e$ is cycle trivial in $\Gamma / e$, then $\Gamma^{\prime}$ is cycle trivial in $\Gamma$.

Suppose $F$ is a surface in the boundary of a 3-manifold $X$, and $\alpha$ a simple closed curve in $F$. We use $X_{\alpha}$ to denote the manifold obtained from $X$ by attaching a 2handle to $X$ along $\alpha$, and use $F_{\alpha}$ to denote the corresponding surface in $X_{\alpha}$. More explicitly, $X_{\alpha}=X \cup_{\varphi}\left(D^{2} \times I\right)$, where $\varphi$ identifies $\partial D^{2} \times I$ to a regular neighborhood $A$ of $\alpha$ in $F$, and $F_{\alpha}=(F-A) \cup\left(D^{2} \times \partial I\right)$. The following result was proved in [11]. When $K=\emptyset$, it reduces to Jaco's Handle Addition Lemma [2, Lemma 1]. In [11] the conclusion was stated as $\left|\partial D^{\prime} \cap K\right| \leq|\partial D \cap K|$, but the proof there has actually given the following stronger version.

Proposition 2.4. Let $F$ be a surface on the boundary of a 3-manifold $X$, and $K a$ 1-manifold in $F$ with $F-K$ compressible in $X$. Let $\alpha$ be a simple loop in $F-K$. If $F_{\alpha}$ has a compressing disk $D$ in $X_{\alpha}$, then $F-\alpha$ has a compressing disk $D^{\prime}$ in $X$ such that $\partial D^{\prime} \cap K \subset \partial D \cap K$.

Lemma 2.5. Let $\Gamma$ be a graph in a 3-manifold $M$ which has no lens space or $S^{1} \times S^{2}$ summand. Suppose $\Gamma$ has only one vertex $v$ and $n$ edges $e_{1}, \ldots e_{n}$. Then $\Gamma$ is cycle trivial if and only if $\partial N\left(\Gamma^{\prime}\right)$ is compressible in $E\left(\Gamma^{\prime}\right)$ for all subgraphs $G^{\prime} \neq v$ of $G$.

Proof. If $n=1$, then $\Gamma$ is a knot in $M$. Let $D$ be a compressing disk of $\partial N(\Gamma)$ in $E(\Gamma)$. If $\partial D$ is not a longitude of $\Gamma$, then $N(\Gamma) \cup N(D)$ is a punctured lens space or punctured $S^{1} \times S^{2}$, contradicting our assumption about $M$. Hence it can be extended to a disk in $M$ bounded by $\Gamma$, so $\Gamma$ is a trivial cycle.

Now assume that $\delta(v)$ is compressible in $E(\Gamma)$. Then a compressing disk can be extended to a sphere $S$ in $M$ intersecting $\Gamma$ only at $v$, with both sides of $S$ containing parts of $\Gamma$. Note that $S$ must be separating because $M$ has no $S^{1} \times S^{2}$ summands. Let $\Gamma^{\prime}, \Gamma^{\prime \prime}$ be the subgraphs of $\Gamma$ in the two sides of $S$. By induction they are cycle trivial, so each cycle $C$ of $\Gamma^{\prime}$ bounds a disk $D$ with interior disjoint from $\Gamma^{\prime}$. Isotop $D$ so that $D \cap S$ is a union of circles which are mutually disjoint except possibly at the point $v$. By doing 2-surgeries along disks in $S$ bounded by innermost circles of $C \cap S$, we can change $D$ to a disk with interior disjoint from $\Gamma^{\prime} \cup S$, and hence disjoint from $\Gamma$. Similarly, every cycle in $\Gamma^{\prime \prime}$ is trivial in $\Gamma$. 
It remains to show that if $n>1$ then $\delta(v)$ is compressible. Let $m_{i}$ be the central curve of the annulus $\delta\left(e_{i}\right)$. Let $F=\partial N(\Gamma)$. Let $K$ be a maximal subset of the $m_{i}$ 's such that $F-K$ is compressible. Notice that if $K$ contains all the $m_{i}$ 's, then we are done because $F-K$ retracts to $\delta(v)$. So assume that $m_{1}$ is not in $K$. Then $F-K$ is compressible in $E(\Gamma)$, but $F-\left(K \cup m_{1}\right)$ is incompressible. After attaching a 2-handle along $m_{1}$, the surface $F_{m_{1}}=\partial N\left(\Gamma-e_{1}\right)$, and the manifold $E(\Gamma)_{m_{1}}=E\left(\Gamma-e_{1}\right)$. By induction we may assume that $\Gamma-e_{1}$ is cycle trivial, so the loop $e_{2}$ is trivial in $\Gamma-e_{1}$. This means that there is a compressing disk $D$ of $F_{m_{1}}$ intersecting $m_{2}$ just once, and is disjoint from the other $m_{i}$ 's, so it intersects $K$ at most once, at a point in $m_{2}$. Applying Proposition 3, we know that $E(\Gamma)$ contains a compressing disk $D^{\prime}$ of $F$ which is disjoint from $m_{1}$, and intersects $K$ at most at one point in $m_{2}$. If $D^{\prime}$ is disjoint from $m_{2}$, then $F-\left(K \cup m_{1}\right)$ is compressible. If $D^{\prime}$ intersects $m_{2}$ at one point, then the boundary of a regular neighborhood of $\partial D^{\prime} \cup m_{1}$ in $F$ is a compressing disk of $F-\left(K \cup m_{1}\right)$. Either case contradicts the assumption that $F-\left(K \cup m_{1}\right)$ is incompressible.

A subgraph $\Gamma^{\prime}$ of $\Gamma$ is said to be essential if it contains some cycles.

Theorem 2.6. Let $\Gamma$ be a graph in a 3-manifold $M$ which has no lens space or $S^{1} \times S^{2}$ summand. Then $\Gamma$ is cycle trivial if and only if $\partial N\left(\Gamma^{\prime}\right)$ is compressible in $E\left(\Gamma^{\prime}\right)$ for all essential subgraphs $\Gamma^{\prime}$ of $\Gamma$.

Proof. By induction on the number of edges we may assume that all subgraphs and quotient graphs of $\Gamma$ are cycle trivial. By the same argument as in the proof of Lemma 2.5 , we see that the theorem is true if each component of $\Gamma$ has just one vertex. Therefore, we may assume that $\Gamma$ has some non loop edges.

Let $C$ be a cycle of $\Gamma$. If there is a non loop edge $e$ of $\Gamma$ which is not on $C$, then by applying Corollary 2.3 to $\Gamma^{\prime}=\Gamma-e$, we see that $C$ is trivial in $\Gamma$. So assume that $C$ contains all the non loop edges of $\Gamma$. If $C$ is a component of $\Gamma$, we may delete the extra vertices on $C$ to reduce to the case that each component of $\Gamma$ has just one vertex. Hence we may assume that there is a loop $e_{1}$ with $\partial e_{1}$ on $C$. By the above, $e_{1}$ is trivial in $\Gamma$, so it bounds a disk $D$ with interior disjoint from $\Gamma$. By induction, $\Gamma-e_{1}$ is trivial, so $C$ bounds a disk $D^{\prime}$ with interior disjoint from $\Gamma-e_{1}$. Now we can isotop $e_{1}$ through disks on $D$ bounded by outermost arcs of $D \cap D^{\prime}$ to eliminate all intersections of $e_{1}$ with $D^{\prime}$. The reverse isotopy moves $D^{\prime}$ to a disk with interior disjoint from $\Gamma$.

Remark. A graph in $M^{3}$ is called planar if it lies on an embedded 2-sphere in $M$. It is abstractly planar if it can be embedded into an abstract 2-sphere. When $M=S^{3}$, 
Lemma 2.5 was proved by Gordon [1]. It was also proved that if $\Gamma$ has two vertices, then it is planar if the exterior of all subgraphs are handlebodies. In [8] J. Simon conjectured that if a graph $\Gamma$ is abstractly planar, then Gordon's theorem is still true, that is, $\Gamma$ is planar if and only if the exteriors of all subgraphs of $\Gamma$ has free fundamental group. Simon and Wolcott [9] proved that this is true if $\Gamma$ is the handcuff or double

$\theta$ graph. The conjecture was fully proved by Scharlemann and Thompson $[4,5,7]$. In [12] it was noticed that if $\Gamma$ is abstractly planar, then it is planar if and only if it is cycle trivial. Using this fact and Theorem 2.1, a new proof of the ScharlemannThompson Theorem was given in [12]. Because of this fact, Theorem 2.6 may be considered as a generalization of the Scharlemann-Thompson Theorem. When $M=$ $S^{3}$, Theorem 2.6 was first proved by Robertson, Seymour and Thomas [3] under the stronger assumption that the exteriors of any subgraph of $\Gamma$ are connected sums of handlebodies. Scharlemann and Thompson [6] have proved a theorem about sliding arcs in handlebodies, which simultaneously generalize the $S^{3}$ version of Theorem 2.6 and Waldhausen's Theorem [10].

\section{REFERENCES}

[1] C. Gordon, On the primitive sets of loops in the boundary of a handlebody, Topology Appl. 27 (1987), 285-299.

[2] W. Jaco, Adding a 2-handle to 3-manifolds: An application to Property R, Proc. Amer. Math. Soc. 92 (1984), 288-292.

[3] N. Robertson, P. Seymour and R. Thomas, Linkless embeddings of graphs in 3-space, Bull. Amer. Math. Soc. 28 (1993), 84-89.

[4] M. Scharlemann, planar graphs, family trees, and braids, preprint (1989).

[5] M. Scharlemann and A. Thompson, Detecting unknotted graphs in 3-space, J. Diff. Geom. 34 (1991), 539-560.

[6] , Pushing arcs and graphs around in handlebodies, preprint.

[7] A. Thompson, A polynomial invariant of graphs in 3-manifolds, Topology 31 (1992), 657-665.

[8] J. Simon, Molecular graphs as topological objects in space, J. Comput. Chem. 8 (1987), 718-726.

[9] J. Simon and K. Wolcott, Minimally knotted graphs in $S^{3}$, Topology and its Appl. 37 (1990), $163-180$.

[10] Waldhausen, Heegaard-Zerlegungen der 3-sphäre, Topology 7 (1968), 195-203.

[11] Y-Q. Wu, A generalization of the handle addition theorem, Proc. Amer. Math. Soc. 114 (1992), $237-242$.

[12] _ On planarity of graphs in 3-manifolds, Comment. Math. Helv. 67 (1992), 635-637. 